\title{
Host Regulators of Liver Fibrosis During Human Schistosomiasis
}

\author{
Severin Donald Kamdem 1,2,3, Roger Moyou-Somo ${ }^{4}$, Frank Brombacher ${ }^{1,2,3,5}$ and \\ Justin Komguep Nono ${ }^{1,2,3,4 *}$ \\ ${ }^{1}$ Cape Town Component, International Centre for Genetic Engineering and Biotechnology, Cape Town, South Africa, \\ ${ }^{2}$ Division of Immunology, Health Science Faculty, University of Cape Town, Cape Town, South Africa, ${ }^{3}$ Immunology of \\ Infectious Diseases Unit, South African Medical Research Center, Cape Town, South Africa, ${ }^{4}$ The Medical Research Centre, \\ Institute of Medical Research and Medicinal Plant Studies, Ministry of Scientific Research and Innovation, Yaoundé, \\ Cameroon, ${ }^{5}$ Faculty of Health Sciences, Wellcome Centre for Infectious Diseases Research in Africa, Institute of Infectious \\ Diseases and Molecular Medicine (IDM), University of Cape Town, Cape Town, South Africa
}

Liver fibrosis is a wound-healing process purposely aimed at restoring organ integrity after severe injury caused by autoimmune reactions, mechanical stress or infections. The uncontrolled solicitation of this process is pathogenic and a pathognomonic feature of diseases like hepatosplenic schistosomiasis where exacerbated liver fibrosis is centrally positioned among the drivers of the disease morbidity and mortality. Intriguingly, however, liver fibrosis occurs and progresses dissimilarly in schistosomiasis-diseased individuals with the same egg burden and biosocial features including age, duration of residence in the endemic site and gender. This suggests that parasite-independent and currently poorly defined host intrinsic factors might play a defining role in the regulation of liver fibrosis, the hallmark of morbidity, during schistosomiasis. In this review, we therefore provide a comprehensive overview of all known host candidate regulators of liver fibrosis reported in the context of human schistosomiasis.

Keywords: liver fibrosis, host factors, human schistosomiasis, hepatosplenic schistosomiasis, wound healing

\section{INTRODUCTION}

Schistosomiasis represents the second most important parasitic disease in the world in terms of public health impact (1). With 120 million symptomatic (2) and 20 million with severe morbidity (3), schistosomiasis results in the loss of 70 million disability-adjusted life years (DALYs) (4) and preferentially occurs in developing countries with almost 200 million infected in Africa alone (5).

Schistosomes cause varying clinical symptoms and organ complications due to the species-specific tropisms of the egg-laying adult worms (6). While Schistosoma haematobium adult worms reside in vessels of the bladder causing urinary schistosomiasis, those of Schistosoma mansoni and Schistosoma japonicum reside in the mesenteric veins of the intestinal tract causing hepatosplenic schistosomiasis leading to progressive liver fibrosis and portal hypertension (6).

The immunology of schistosomiasis has progressed through the use of murine and non-human primate animal models, corroborating in many cases with human data $(7,8)$. The disease morbidity is driven by schistosome eggs and not directly by adult worms (9). Upon infection, a fraction of the produced eggs is not excreted by the host and become permanently lodged in organs such as the intestines and liver (S. mansoni and S. japonicum) or in the bladder and urogenital system (S. haematobium). Highly immunogenic and cytolytic substances are released within the egg secretions (10). This induces a granulomatous and fibrotic response from the host largely characterized by T helper-2 cytokines (IL-4, IL-5, and IL-13), eosinophils and alternatively activated macrophage 
$(11,12)$. Host granulomas primarily form to contain and wall off the trapped toxic eggs products (13), which allows the host to live with the infection for many years. However, detrimental effects associated with persistent granulomas such as excessive collagen deposition leading to untoward fibrosis and portal hypertension arise, causing host pathology (13).

\section{LIVER FIBROSIS DURING HUMAN SCHISTOSOMIASIS}

The liver is an organ made by many specialized resident non-parenchymal cells, including Kupffer cells, liver sinusoidal endothelial cells and hepatic stellate cells (HSCs). First described by Kupffer in 1876 (14), HSCs which represent $5-8 \%$ of all liver cells (15) are located in the space of Disse in the liver sinusoid (16). These cells are responsible for maintenance of the extracellular matrix, storage of vitamin A (17), with a possible role in controlling blood flow through the liver (18). Upon stimulation, in response to injuries, normally quiescent HSCs (qHSCs) are activated HSCs (aHSCs), lose their ability to store vitamin $A$, increase expression of alpha-smooth muscle actin $(\alpha$-SMA), a profibrotic gene, and develop a broader "stretched" cytoplasm. aHSCs then undergo a process of trans-differentiation toward collagen-producing liver myofibroblasts, the main cell type responsible for hepatic fibrosis (19).

Hepatic fibrosis comes from a variety of etiologies including hepatosplenic schistosomiasis (20), following the accumulation of parasitic eggs within the liver (21). Adult pairs of S. mansoni worms, particularly, reside within the mesenteric veins where females release on average 340 eggs per female per day with rates ranging between 190 and 658 eggs depending of the strain; this rate is higher for S. japonicum (22). More than 50\% of eggs are carried to the liver by the portal circulation where they become trapped in the liver sinusoids (13). This is associated with the production of profibrotic cytokines $(23,24)$. Both murine and human infections with $S$. japonicum or S. mansoni reveal aHSCs as the key drivers of hepatic fibrosis $(25,26)$.

In the context of hepatosplenic schistosomiasis, an accumulating number of studies have reported disparities between prevalence of infection and levels of tissue morbidity, characterized by the stage of advancement of hepatic fibrosis and the presence/absence of periportal fibrosis (11, 27-30). For example, despite a higher prevalence and intensity of $S$. mansoni infections, in Kenya $(31,32)$ and Mali (33) compared to Egypt $(31,32)$ and Sudan $(34,35)$, the prevalence of periportal fibrosis in endemic sites is considerably higher in the latter countries. Furthermore, even adjacent communities with comparable levels of S. mansoni infection, exhibit considerable differences in their prevalence of periportal fibrosis (11). Although some possible explanations for these observed differential morbidity patterns could well be the different duration, intensity of infection $(32,35)$, the host genetic background $(36,37)$ has been increasingly suggested as a central basis for these discrepancies $(11,30,38-42)$ as it appears that hepatic fibrosis occurs and progresses dissimilarly in schistosomiasis-diseased individuals with the same extrinsic and biosocial risk factors. In fact, in another recent study conducted in a village of rural Cameroon endemic for hepatosplenic schistosomiasis, participants with similar egg excretion profiles, similar age/gender distribution, same length of residence in the area, same frequency and duration of daily exposure to contaminated water, same social status and within similar dates of reinfection (i.e., previously treated with Praziquantel at the same time) as judged by the average number of eggs excreted in the feces, displayed a strikingly different stage of advancement of schistosomiasisdriven hepatic fibrosis (43). This indicated that, although the presence of the parasite eggs prompt the onset of liver lesions, host factors might indeed play a defining role in the regulation of liver fibrosis during hepatosplenic schistosomiasis. With the well documented and successful concept of host-directed therapies against diseases now including infectious diseases $(37,39,42,44,45)$, the recollection of known host-derived factors that potentially play a role in the control of liver fibrosis during human schistosomiasis (pro- or anti-fibrotic) is long overdue for research on schistosomiasis in particular and all fibroproliferative diseases in general.

\section{HUMANS FACTORS ASSOCIATED WITH LIVER FIBROSIS DURING SCHISTOSOMIASIS}

The present compilation of host factors reported to associate with the progression/resistance to hepatic fibrosis during Human hepatosplenic schistosomiasis (Table 1 and Figure 1) should facilitate subsequent investigations aimed at validating their role as hepatic fibrosis monitoring tools and for the design of hostdirected intervention strategies against morbidity due to hepatic fibrosis during schistosomiasis.

\section{Cytokines and Cytokine Receptors}

Abundance of information from murine studies links type 2 cytokines (Th2) rather than Type 1 (Th1) responses with the development of hepatic fibrosis $(80,81)$. These studies have defined a dominant role for Th2 immune mediators in the liver fibrotic process during hepatosplenic schistosomiasis $(80,82)$. As a telling example, a recent finding in mechanistic chronic murine schistosomiasis model, has demonstrated an amelioration of liver fibroproliferative pathology following the transgenic reduction of IL-4 receptor alpha-mediated signaling (81). A parallel is now emerging in human studies. High levels of IL-4 and IL13 (secreted primarily by type- 2 immune cells such as Th2 cells to act toward the alternatively activation of macrophages into M2 and the activation of Hepatic stellate cells, Figure 1) were found to associate with periportal fibrosis progression during schistosomiasis in infected individuals from Brazil, Bahia, Philippines and Zambia (42, 46, 47, 49, 51). In addition, an IL13 promoter enhancing single nucleotide polymorphism (SNP), rs1800925, has been shown to strongly associate with a higher risk of pathological hepatic fibrosis in S. japonicum-infected individuals (50).

Another type-2 cytokine receptor, ST2 in its soluble form (sST2), has been reported to be present at high level in the serum 
TABLE 1 | Reported Pro and anti-fibrotic human host factors

during hepatosplenic schistosomiasis.

\begin{tabular}{lc}
\hline PROFIBROTIC vs. ANTI-FIBROTIC & \\
\hline Profibrotic factors & References \\
\hline Type 2 Cytokines (IL-4; IL-5 and IL-13) & $(41,42,46-51)$ \\
Interleukin 33 Receptor (ST2) & $(52)$ \\
Tumor Necrosis Factor alpha (TNF- $\alpha$ ) & $(11,30,44,49,53-55)$ \\
CCL3 & $(56)$ \\
CCL24/sTNFR1/MIF & $(57)$ \\
Connective Tissue Growth Factor (CTGF) & $(39,58-60)$ \\
Transforming Growth Factor-beta 1 (TGF- 31$)$ & $(61)$ \\
Vascular Endothelial Growth Factors (VEGF) & $(62,63)$ \\
Hedgehog ligand (Hh) & $(64)$ \\
Osteopontin (OPN) & $(65-67)$ \\
Antibodies (IgG4 and IgE) & $(68-71)$ \\
Eosinophils/Eosinophil cationic protein (ECP) & $(49,72)$ \\
Mannose-Binding Lectin (MBL) & $(73)$ \\
MicroRNAs & $(74)$ \\
High mobility group box 1 (HMGB1) & $(75)$ \\
\hline Anti-fibrotic factors & References \\
\hline Interferon gamma (INF-y) & $(8,11,30,36,37,40)$ \\
Interleukin 6 (IL-6) & $(51)$ \\
Interleukin 10 (IL-10) & $(11,40,45,51)$ \\
Chemokines: RANTES (CCL5) & $(11)$ \\
Major Histocompatibility Class II (MHC II) & $(76-78)$ \\
Regulatory T cells (Tregs) & $(79)$ \\
\hline
\end{tabular}

of diseased patients with advanced hepatic fibrosis during $S$. japonicum infection (52).

In sharp contrast, the canonical Th1 cytokine INF- $x$ has been associated with fibrosis reduction during human schistosomiasis. In Sudanese patients, higher levels of INF-y were associated with a marked reduction of the risk of fibrosis during schistosomiasis $(11,30)$. Additionally, lower levels of INF-y were linked to a higher risk (more than 8-fold comparing to people with large amount of INF-y) of periportal fibrosis in patients from China and Uganda $(11,40)$. Further investigations at a molecular level revealed that a polymorphism in the INF-rreceptor 1 (IFNGR1) gene which lead to reduced functionality of the receptor, may account for increased susceptibility to severe fibrosis (8). In addition, INF-y gene SNP, translating into reduced gene transcription, associated with more severe fibrosis in Sudanese patients $(8,36,37)$ supporting a protective action of INF-x against hepatic fibrosis during schistosomiasis. These reports are consistent with the powerful antifibrogenic role of INF-y (primary product of type-1 NK and Th1 cells and acting predominantly on macrophages to drive M1 differentiation) which inhibit the transdifferentiation of HSCs into myofibroblast, reduces the production of extracellular matrix proteins and increases the collagenase activity of the liver (83). Finally, IL-6 (secreted by pro-inflammatory cellular responses such as that of classically activated macrophages M1 to counter HSC activation) was also claimed to be protective against severe fibrosis by the team of Mutengo (51) who demonstrated that IL-6 was expressed considerably more by S. mansoni eggstimulated whole blood cultures from individuals with no or mild fibrosis when compared to cultures of individuals with moderate/severe fibrosis (51). This is consistent with reports that associate the lack of IL- 6 with increase in liver injury (84) and the recent potential therapeutic ability of IL- 6 to reverse liver fibrosis (85).

Intriguingly, as another Th1-related cytokine, Tumor necrosis alpha (TNF- $\alpha$ ) at higher levels in supernatants from patient's whole-blood cultures, rather strongly associated with an increased risk of periportal fibrosis during hepatosplenic schistosomiasis $(11,30,49,53)$. In fact, the TNF- $\alpha$ 308.2 SNP or rs1800629(AA), translating into an increased transcription of the TNF- $\alpha$ gene (55), has been shown to correlate with a more severe hepatic fibrosis during S. mansoni infections (54). The picture is a clearly more nuanced as a study in Uganda observed a differential association between INF- $\gamma$, TNF- $\alpha$ and fibrosis (86) where TNF- $\alpha$ reportedly acted by balancing the protective effect of INF-x (87); a trend confirmed in Zambia (51). Overall, adding to observations in experimental models, a strong case is made for INF- $x$ as an anti-fibrotic cytokine $(11,30)$, whereas TNF- $\alpha$ (also produced by Th1 cells and macrophages) primarily appears to aggravate hepatic fibrosis during schistosomiasis (87).

Reports on the possible role of IL-10 (secreted by regulatory cells such as regulatory myeloid and/or $\mathrm{T}$ cells) in relation to hepatic fibrosis made mention of a regulatory role of the il-10 gene SNPs on the progression of periportal fibrosis (45). In fact, higher risk of severe periportal fibrosis was reported when IL-10 production by blood mononuclear cells from schistosomiasisdiseased patients was low $(11,40,51)$ suggesting a protective role of IL-10 against hepatic fibrosis. The anti-inflammatory and immunosuppressive potential of IL-10 which regulate the inflammatory process, central to tissue fibrosis, could be an explanation for these observations (88).

Consequently, the interplay between the arms of the immune response, as defined by the host overall cytokine profile, controls the inflammatory response and determines the evolution of liver fibrosis during schistosomiasis.

\section{Chemokines}

In the last decades, many studies have shown that tissue injury leads to the production of chemokines that orchestrate cell trafficking to the site of injury during infectious diseases (89). In fact, peripheral blood mononuclear cells from schistosome-infected patients with ultrasonographicallydefined hepatosplenomegaly produced high levels of CCL3 when exposed to egg antigen; and marked them as group under greater risk of developing severe disease with hepatic fibrosis (90). This observation was corroborated by independent data obtained from murine models which reported a less severe granulomatous response in CCL3-deficient mice following schistosome infection $(56,90)$. The findings were further confirmed in another study where the plasma concentrations of CCL3 and that of other chemokines, namely CCL24, sTNFR1 and MIF (migration inhibitory Factor), were associated with hepatic fibrosis progression (57). 


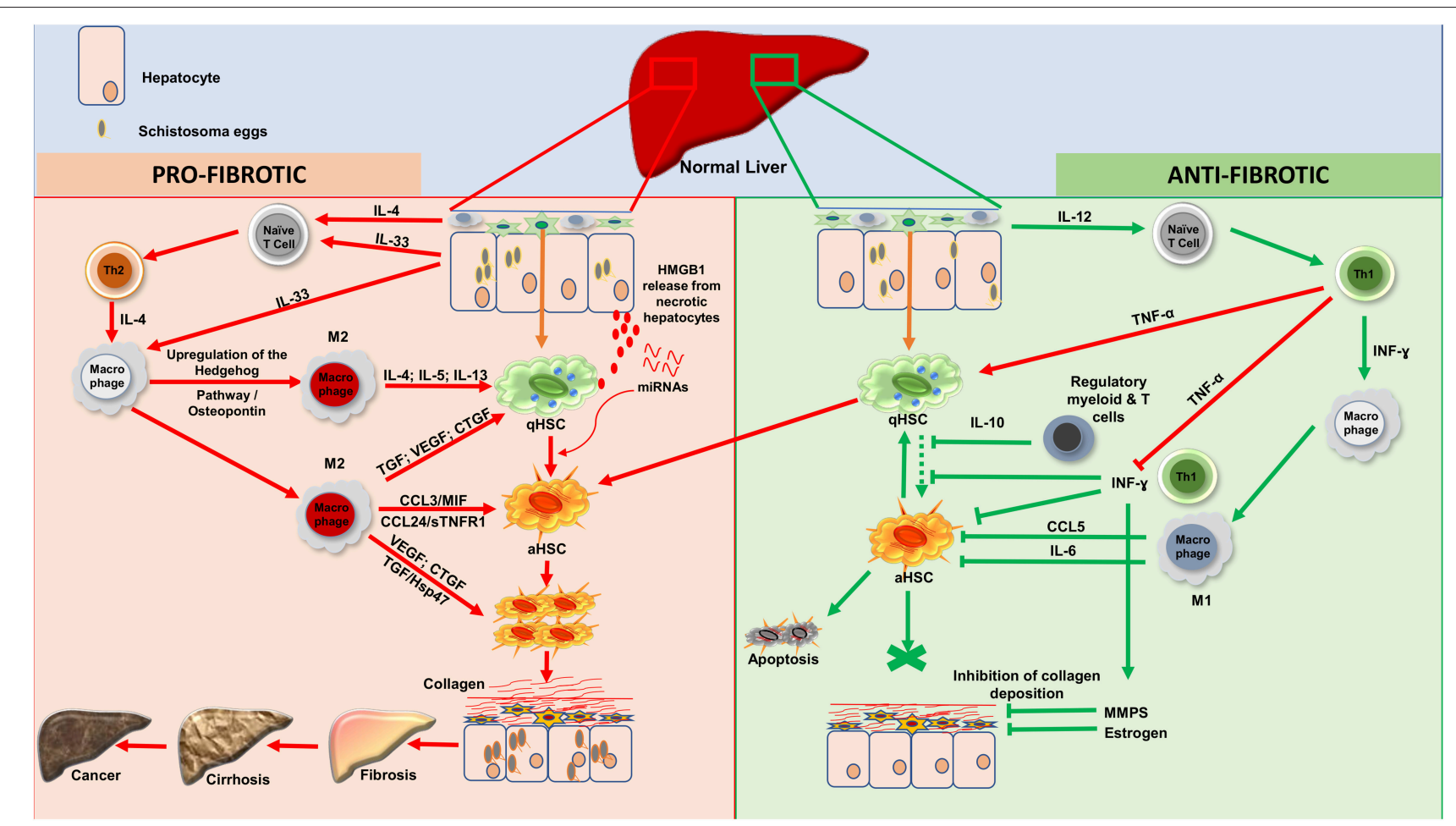

FIGURE 1 | Processes of liver fibrosis progression and regression during human hepatosplenic schistosomiasis. Profibrotic processes: Upon stimulation, by Schistosoma spp. eggs antigens, injured hepatocytes will release IL-33 as a danger alerting molecule. Moreover, schistosome eggs will drive, via cells such as Innate lymphoid cells, the release of IL-4. IL-33 and IL-4 will act on naiive T cells to promote Th2 differentiation. IL-33 will also induce through his receptor ST2 located on macrophages, the upregulation of the Hedgehog pathway which will then drive via osteopontin the alternative activation of macrophages (M2). The effect of IL-33 on endothelial cells will also promote angiogenesis which will increase the vascular permeability. With the support of TNF- $\alpha$ produced by Th1, Th2 cells will then produce large amount of type 2 cytokines (IL-4, IL-5, and IL-13) which will induce the activation of qHSCs. M2 differentiated under the flow of Th2-released type-2 cytokines can also foster the activation of QHSCs by releasing TGF- $\beta 1$, VEGF or CTGF. While the two latter can directly stimulate the proliferation of aHSCs, TGF- $\beta 1$ can act through the Hsp47. This action is sustaining by some chemokines especially (CCL3; MIF; CCL24 and sTNFR1). Moreover, necrotic hepatocytes might release High mobility group box 1 (HMGB1) protein as an alarmin that will ultimately foster HSC activation. In the midst of advanced fibrosis, human livers might further release miRNAs that alter host metabolism, triggering HSC activation to promote tissue fibrosis. Altogether, proliferating aHSCs will produce a very large amount of collagen, which will accumulate and lead to fibrosis which can progress to cirrhosis and then ultimately to liver cancer. Anti-fibrotic processes: Three possibilities can account for regression of fibrosis: apoptosis; senescence or reversion of aHSCs to their quiescent stage. Upon stimulation, hepatic resident macrophages will produce IL- 12 which will drive the differentiation of Naiive T-cell to Th1. Th1 cells will release INF-y which will classically activate macrophages (M1). Various regulatory T and/or myeloid cells are also known to produce IL-10 which can block the activation of qHSCs to consolidate an anti-fibrotic effect. This action is further supported by the large amount of INF-r produced by Th1 although balanced by the level of secreted TNF- $\alpha$. aHSCs might return to the quiescent/senescent state under the action of IL-6; CCL5 or INF-y or undergo apoptosis depending on how strong the profibrotic signal was. INF-y can also activate MMPs to digest the deposited collagen and this action is reinforced by estrogen in women. IL, Interleukin; HSCs, Hepatic Stellate Cells; qHSCs, quiescent Hepatic Stellate Cells; aHSCs, activated Hepatic Stellate Cells; TGF- $\beta 1$, Transforming Growth Factor beta 1; VEGF, Vascular Endothelial Growth Factor; CTGF, Connective Tissue Growth Factor; MIF, Migration Inhibitory Factor; INF-x, Interferon gamma; TNF- $\alpha$, Tumor Necrosis Factor alph; MMPS, Matrix Metalloproteinase; sTNFR1, Soluble Tumor Necrosis Factor Receptor-1; CCL3, Chemokine C-C motif ligand 3; CCL5, Chemokine C-C motif ligand 5; CCL24, Chemokine C-C motif ligand 24; M1, Classical activated Macrophages; M2, Alternatively activated Macrophages; Hsp47, Heat Shock Protein 47; Th1, Type 1 Lymphocytes; Th2, Type 2 Lymphocytes. HMGB1, High mobility group box 1. NB, These 2 cascades of processes take place simultaneously during the host attempt to heal the wound after injury by trapped schistosome eggs.

RANTES (CCL5), another monocyte/macrophage-derived chemokine which has a central role in the regulation of both Th1 and Th2 responses by controlling the migration and activation of leukocytes on the site of inflammation (91), was found to negatively associate with periportal fibrosis (11).

Overall, these observations are consistent with published reports that showed that chemokines affect several processes, including differentiation and activation of fibroregulatory cells including fibroblasts which are central to the onset and progression of tissue fibrosis (56).

\section{Growth Factors}

Many Growth factors are known to be fibrogenic and thus contribute to hepatic fibrosis in schistosome infections independently or in association with other factors (23). Dessein et al. in a primary study reported that several SNPs (rs9402373, rs12526196, rs9402373, and rs1256196) that lie close to the Connective Tissue Growth Factor (CTGF) gene are associated with severe hepatic fibrosis in individuals affected by hepatosplenic schistosomiasis $(39,60)$. This observation gains support from the observation that CTGF overproduction, by cells such as M2 macrophages, was proposed to drive tissue 
fibrosis (61). In fact, CTGF has now been shown to promote fibroblast proliferation, migration, adhesion, and extracellular matrix formation $(58,59)$. Notably, CTGF is mostly upregulated by TGF- $\beta 1$ (23), a well-known fibrogenic growth factor (also secreted by M2 macrophages) which was also identified to be positively associated through the heat shock protein 47 (Hsp47) with hepatic fibrosis in Chinese patients infected with S. japonicum (61).

Apparent controversial results were found for the implication of Vascular Endothelial Growth Factor (VEGF) in periportal fibrosis. Egyptian patients with periportal fibrosis had significantly elevated serum levels of VEGF (again secreted by cells such as M2 macrophages) when compared to nondiseased controls (62) whereas no significance difference in serum levels of this factor was reported in Brazilian cohorts of hepatosplenic schistosomiasis patients with or without hepatic fibrosis (63). This difference could be attributed to the small sample size used in the latter study (28 participants vs. 90 in the Egyptian study) and the heterogeneity in the case and control groups. Conclusively, a case is maintained for the clinical evidence that circulating levels of VEGF correlate with hepatic fibrosis progression considering its known role as regulator of angiogenesis, a crucial step in the process of fibrosis (62).

\section{Hedgehog Ligands/Osteopontin}

Macrophages when activated via the alternative pathway, are instrumental in the liver fibrosis process (92). Understandably, Hedgehog ( $\mathrm{Hh}$ ) ligands, produced by macrophages were found to promote hepatic fibrosis during schistosomiasis by a reverse feedback action leading to an alternative activation of macrophages (64). This is consistent with the now defined role of the Hedgehog pathway in the onset and progression of vascular remodeling, regulation of HSC activation thus schistosomiasisrelated fibrosis (64). In fact, Syn et al. elegantly demonstrated that the pro-fibrogenic effects induced by the Hedgehog pathway are mediated by a secreted host phosphoprotein, osteopontin (93). This is substantiated by the observation that high osteopontin level in serum and liver tissue correlates with hepatic fibrosis during schistosome infection $(65,66)$. This correlation was further validated in mechanistic chronic murine schistosomiasis model where higher levels of osteopontin release by macrophages associated with hepatosplenic disease $(65,66)$. Fundamentally, these findings are consistent with the demonstrated role of osteopontin in inflammation, immunity, angiogenesis, fibrogenesis and carcinogenesis in various tissues (94).

\section{Antibodies (IgG4 and IgE)}

The isoform 4 of the Immunoglobulin class G (IgG4) was found 20 times elevated in patients with chronic schistosomiasis compared to non-infected controls (71). A role for IgG4 in schistosome-driven liver fibrosis was more elegantly defined as association was demonstrated between the former and liver fibrosis following schistosome infection (68-70). These observations were extended to Immunoglobulin E ( $\operatorname{IgE}$ ) which was also associated, although to a lower extent, to disease severity $(69,70)$. Together, these studies have defined, pending in-depth empirical studies, a possible role for host Immunoglobulins, of the G4 and E types, in the fibrotic process resulting from human schistosome infections (70).

\section{Eosinophil Markers and Cation Protein}

Other immune cells do also participate by their products in the process of hepatic fibrosis during hepatosplenic schistosomiasis. Eosinophils which play an important protective role in the immune response to $S$. mansoni infection (95), upregulate activation-related surface markers (CD69 and CD23) in response to host-released Type 2 cytokines, which altogether selectively associate with periportal fibrosis progression during hepatosplenic schistosomiasis. Moreover, Eosinophil Cationic Protein (ECP), a protein secreted by Eosinophils that efficiently kills $S$. mansoni at the larval stage, also affect fibroblast function. Eriksson et al. (72) identified the ECP 434GG genotype, which translate into a higher cytotoxic potential (96) as being associated with periportal fibrosis during hepatosplenic schistosomiasis (72). These observations are consistent with the suggested role of eosinophils in wound-healing, remodeling, development of post inflammatory fibrosis $(72,97)$ and the association of tissue eosinophilia and eosinophil degranulation with several other fibrotic syndromes (98). Moreover, human eosinophils express all isoforms of the Transforming Growth Factor beta (TGF- $\beta 1,2,3)(99)$ hinting to a possible role in the liver fibrosis process during human hepatosplenic schistosomiasis.

\section{Mannose-Binding Lectin (MBL)}

In the northeastern of Brazil, Mannose-Binding Lectin which also acts as an opsonin released by mononuclear innate cells (monocytes, macrophages, dendritic cells), was reported to be a risk factor for advanced periportal fibrosis in chronic hepatic diseases as well as S. mansoni infection (73). This can be explained by the role of MBL as a central component of the innate immune response and its highly collagenous activity following host infection by invading pathogens (100).

\section{HLA Class II}

Positive and negative associations between HLA class II alleles and the risk of individuals developing moderate to severe hepatic fibrosis following $S$. japonicum infection were reported. While alleles HLA-DRB1*1501; HLA-DQB1*0601; DRB1*11011, DRB1*0409, and DRB1*0701 were associated with resistance to hepatic fibrosis; some other HLA-DRB1 alleles (HLADRB1*0901; HLA-DRB1*1302; DRB1*1202, DRB1*1404, and DRB1*1405) and Two HLA-DQB1 alleles (HLA-DQB1*0303 and HLA-DQB1*0609) were in contrast significantly associated with rapid progression of hepatic fibrosis $(77,78)$. In addition, HLA$\mathrm{DPA}^{*} 0103$ and DPB1*0201 haplotypes were also associated with protection from both moderate and severe fibrosis (76).

\section{MicroRNAs}

Some microRNAs were reported for the first time through miRNome and transcriptome analyses of livers from humans infected with Schistosoma japonicum in China, to play an important role in schistosomiasis-driven hepatic fibrosis. Notably, hsa-miR-150-5p, hsa-miR-10a-5p, hsa-miR-199a-3p, hsa-miR-4521, hsa-miR-222/221, hsa-miR-663b, and hsa-miR143-3p were found to be upregulated in schistosome-infested 
fibrotic livers (74). These observations could be explained by the defined role of some miRNAs (hsa-miR-222/221 and hsamiR-10a), respectively, reported to drive the activation of HSCs (101), or to upregulate the expression of TGF- $\beta$, a profibrotic growth factor (102); thus corroborating accumulating studies which report predominantly on an upregulation of fibrogenic microRNAs during schistosomiasis $(103,104)$.

\section{Regulatory T Cells}

Very few studies have focused on the role of regulatory $\mathrm{T}$ cells (Treg cells) on liver fibrosis during schistosomiasis. Notwithstanding, in the Hunan province China, high blood Tregs level was associated with severe hepatic fibrosis caused by Schistosoma japonicum (79). This observation aligned with another study which linked a high blood Tregs level with S. mansoni infection (105). A possible explanation for these observations is the clear anti-inflammatory thus anti-fibrotic role of Tregs that accumulate in the blood but failed to be recruited to the liver site of fibrogranulomatous inflammation during schistosomiasis. In fact, a decreased expression of tissue homing markers was reported on Tregs found in the blood of patients with severe fibrosis during schistosomiasis (79). Consequently, a deficit of recruitment of Treg cells to the fibrotic tissue, but rather the accumulation of these Treg cells in the blood, would explain the aggravated tissue fibrotic profile that coincides with elevated blood Tregs during schistosomiasis-driven severe liver fibrosis. An anti-fibrotic role for the host Treg cells is therefore suggested during human hepatosplenic schistosomiasis.

\section{High Mobility Group Box 1 (HMGB1)}

Originally described as a nuclear protein (106), emerging studies have showed that HMGB1 is closely associated with fibrotic disorders, including liver fibrosis (107). In this regard, a study conducted in Brazil reported high levels of HMGB1 in the plasma of both acute and chronically infected-patients with $S$. mansoni when compared with the sera of healthy donors (75). This was further supported in a study highlighting a significantly increased level of HMGB1 in patients with liver fibrosis caused by hepatitis B (108). Additionally, a former study reported that HMGB1 up-regulates alpha-smooth muscle actin ( $\alpha$-SMA) expression in HSCs and then drive fibrosis (109). This trend gained further strength in animal-model-based study where a HMGB1 inhibitor was able to downregulate the production of the profibrotic cytokines IL4, IL-5, and IL-13 and significantly reverse (over $50 \%$ ) liver fibrosis (75).

\section{CONCLUDING REMARKS}

In conclusion, hepatic fibrosis is a very complex process that involves biosocial, genetic, cellular and soluble mediators. Most of these host-derived actors generally come into play as a response to the infection in order to protect the host but progressively drive the substance of the hepatosplenic schistosomiasis morbidity when overly solicited. It is now clear from experimental models and human studies that the expression or lack of expression of such host factors centrally regulate this process. While the factors reported in the present review were identified as independent players (Table 1), the regulation of hepatic fibrosis progression during hepatosplenic schistosomiasis is most likely multifactorial (Figure 1) and will necessitate more studies incorporating this concept. With the alarmingly limited efficiency of mass deworming campaigns alone to eliminate the disease and control the morbidity in resource-limited areas (43), a comprehensive assessment of these host factors is desperately needed as they constitute an attractive avenue for novel disease control strategies. To be used as drug targets, reported human host factors should be validated in animal models to evaluate their potential for therapeutic development. In fact, the targeting of identified profibrotic cytokines (IL-5; IL13) and growth factors (CTGF; TGF- $\beta 1$ and VEGF) should be pursued in conjunction or as an alternative to current disease control measures to attempt to alleviate the fibrosis process during hepatosplenic schistosomiasis. Other attractive candidate targets such as miRNAs should also be targeted, with the hope of reducing HSC proliferation, contractility and suppress angiogenesis by indirect mitigation of the TGF- $\beta$ and VEGFmediated signaling nodes. Encouragingly, this targeting approach is now being undertaken for some suspected profibrotic factors with promising results $(75,81)$. Complementarily, given the advancement of high-throughput analysis tools such as nextgeneration sequencing, the comprehensive identification and clinical validation of host factors that might regulate hepatic fibrosis individually and/or collectively in some schistosomiasisdiseased individuals should be further pursued to provide more candidates for therapeutic evaluation.

\section{AUTHOR CONTRIBUTIONS}

JN and SK defined the topic and designed the search strategy. SK gathered and read articles, as well as wrote the first draft of the manuscript under the supervision of JN. JN corrected and validated the manuscript. JN, SK, FB, and RM-S did a thorough revision of the manuscript. All authors read and approved the final manuscript.

\section{FUNDING}

This project is part of the EDCTP2 programme supported by the European Union, through grant number TMA2016CDF1571 to JN. FB is supported by the International Centre for Genetic Engineering and Biotechnology, the South African National Research Foundation and Medical Research Council. RM-S received funding from the International Centre for Genetic Engineering and Biotechnology (ICGEB) collaborative research grant CRP/CMR15-05. SK is a recipient of a $\mathrm{PhD}$ fellowship from the International Centre for Genetic Engineering and Biotechnology (ICGEB).

\section{ACKNOWLEDGMENTS}

We would like to thank Mary Shelton from UCT Medical Campus Library for the help in elaborating the search strategy. We are grateful to Dr. Jean Jacques Noubiap for helpful discussions during the conception of this review project. 


\section{REFERENCES}

1. Tucker MS, Karunaratne LB, Lewis FA, Freitas TC, Liang YS. Schistosomiasis. Curr Protoc Immunol. (2013) 103:Unit 19.1. doi: 10.1002/0471142735.im1901s103

2. Chitsulo L, Loverde P, Engels D. Schistosomiasis. Nat Rev Microbiol. (2004) 2:12-3. doi: 10.1038/nrmicro801

3. Crompton D. How much human helminthiasis is there in the world? $J$ Parasitol. (1999) 85:397-403. doi: 10.2307/3285768

4. King $\mathrm{CH}$, Dickman $\mathrm{K}$, Tisch DJ. Reassessment of the cost of chronic helmintic infection: a meta-analysis of disability-related outcomes in endemic schistosomiasis. Lancet (2005) 365:1561-9. doi: 10.1016/S0140-6736(05)66457-4

5. Steinmann P, Keiser J, Bos R, Tanner M, Utzinger J. Schistosomiasis and water resources development: systematic review, meta-analysis, and estimates of people at risk. Lancet Infect Dis. (2006) 6:411-25. doi: 10.1016/S1473-3099(06)70521-7

6. Anthony B, Allen JT, Li YS, McManus DP. Hepatic stellate cells and parasite-induced liver fibrosis. Parasit Vectors (2010) 3:60. doi: 10.1186/1756-3305-3-60

7. Abath FG, Morais CN, Montenegro CE, Wynn TA, Montenegro SM. Immunopathogenic mechanisms in schistosomiasis: what can be learnt from human studies? Trends Parasitol. (2006) 22:85-91. doi: $10.1016 /$ j.pt.2005.12.004

8. Dessein A, Kouriba B, Eboumbou C, Dessein H, Argiro L, Marquet S, et al. Interleukin-13 in the skin and interferon-gamma in the liver are key players in immune protection in human schistosomiasis. Immunol Rev. (2004) 201:180-90. doi: 10.1111/j.0105-2896.2004.00195.x

9. Burke ML, Jones MK, Gobert GN, Li YS, Ellis MK, McManus DP. Immunopathogenesis of human schistosomiasis. Parasite Immunol. (2009) 31:163-76. doi: 10.1111/j.1365-3024.2009.01098.x

10. Fairfax K, Nascimento M, Huang SC-C, Everts B, Pearce EJ. (eds). Th2 responses in schistosomiasis. Semin Immunopathol. (2012) 34:863-71. doi: 10.1007/s00281-012-0354-4

11. Booth M, Mwatha JK, Joseph S, Jones FM, Kadzo H, Ireri E, et al. Periportal fibrosis in human Schistosoma mansoni infection is associated with low IL-10, low IFN-gamma, high TNF-alpha, or low RANTES, depending on age and gender. J Immunol. (2004) 172:1295-303. doi: 10.4049/jimmunol.172.2.1295

12. McGonigle S, Beall MJ, Pearce EJ. Eukaryotic initiation factor 2 alpha subunit associates with TGF beta receptors and 14-3-3 epsilon and acts as a modulator of the TGF beta response. Biochemistry (2002) 41:579-87. doi: $10.1021 /$ bi011407z

13. Wynn TA, Thompson RW, Cheever AW, Mentink-Kane MM. Immunopathogenesis of schistosomiasis. Immunol Rev. (2004) 201:156-67. doi: 10.1111/j.0105-2896.2004.00176.x

14. Wake K. "Sternzellen" in the liver: perisinusoidal cells with special reference to storage of vitamin A. Am J Anatomy (1971) 132:429-62. doi: 10.1002/aja.1001320404

15. Geerts A. History, heterogeneity, developmental biology, and functions of quiescent hepatic stellate cells. Semin Liver Dis. (2001) 21:311-35. doi: 10.1055/s-2001-17550

16. Puche JE, Saiman Y, Friedman SL. Hepatic stellate cells and liver fibrosis. Compr Physiol. (2013) 3:1473-92. doi: 10.1002/cphy.c120035

17. Blomhoff R, Wake K. Perisinusoidal stellate cells of the liver: important roles in retinol metabolism and fibrosis. FASEB J. (1991) 5:271-7. doi: 10.1096/fasebj.5.3.2001786

18. Reynaert H, Thompson MG, Thomas T, Geerts A. Hepatic stellate cells: role in microcirculation and pathophysiology of portal hypertension. Gut (2002) 50:571-81. doi: 10.1136/gut.50.4.571

19. Friedman SL. Molecular regulation of hepatic fibrosis, an integrated cellular response to tissue injury. J Biol Chem. (2000) 275:2247-50. doi: $10.1074 /$ jbc.275.4.2247

20. Poelstra K. Liver fibrosis in 2015: crucial steps towards an effective treatment. Nat Rev Gastroenterol Hepatol. (2016) 13:67-8. doi: 10.1038/nrgastro.2015.224

21. Zoni AC, Catala L, Ault SK. Schistosomiasis prevalence and intensity of infection in Latin America and the Caribbean countries, 1942-2014: a systematic review in the context of a regional elimination goal. PLoS Negl Trop Dis. (2016) 10:e0004493. doi: 10.1371/journal.pntd.0004493
22. Loker ES. A comparative study of the life-histories of mammalian schistosomes. Parasitology (1983) $87(\mathrm{Pt} \quad 2): 343-69$. doi: $10.1017 /$ S0031182000052689

23. Leask A, Abraham DJ. TGF-beta signaling and the fibrotic response. FASEB J. (2004) 18:816-27. doi: 10.1096/fj.03-1273rev

24. Wickert L, Steinkruger S, Abiaka M, Bolkenius U, Purps O, Schnabel C, et al. Quantitative monitoring of the mRNA expression pattern of the TGF-betaisoforms (beta 1, beta 2, beta 3) during transdifferentiation of hepatic stellate cells using a newly developed real-time SYBR Green PCR. Biochem Biophys Res Commun. (2002) 295:330-5. doi: 10.1016/S0006-291X(02)00669-1

25. Carson JP, Ramm GA, Robinson MW, McManus DP, Gobert GN. Schistosome-induced fibrotic disease: the role of hepatic stellate cells. Trends Parasitol. (2018) 34:524-40. doi: 10.1016/j.pt.2018.02.005

26. Seki E, Brenner DA. Recent advancement of molecular mechanisms of liver fibrosis. J Hepatobiliary Pancreat Sci. (2015) 22:512-8. doi: 10.1002/jhbp.245

27. Marquet S, Abel L, Hillaire D, Dessein A. Full results of the genomewide scan which localises a locus controlling the intensity of infection by Schistosoma mansoni on chromosome 5q31-q33. Eur J Hum Genet. (1999) 7:88-97. doi: 10.1038/sj.ejhg.5200243

28. Mwatha JK, Kimani G, Kamau T, Mbugua GG, Ouma JH, Mumo J, et al. High levels of TNF, soluble TNF receptors, soluble ICAM-1, and IFN-gamma, but low levels of IL-5, are associated with hepatosplenic disease in human schistosomiasis mansoni. J Immunol. (1998) 160:1992-9.

29. Abel L, Demenais F, Prata A, Souza AE, Dessein A. Evidence for the segregation of a major gene in human susceptibility/resistance to infection by Schistosoma mansoni. Am J Hum Genet. (1991) 48:959-70.

30. Henri S, Chevillard C, Mergani A, Paris P, Gaudart J, Camilla C, et al. Cytokine regulation of periportal fibrosis in humans infected with Schistosoma mansoni: IFN-gamma is associated with protection against fibrosis and TNF-alpha with aggravation of disease. J Immunol. (2002) 169:929-36. doi: 10.4049/jimmunol.169.2.929

31. Blanton RE, Salam EA, Kariuki HC, Magak P, Silva LK, Muchiri EM, et al. Population-based differences in Schistosoma mansoni- and hepatitis C-induced disease. J Infect Dis. (2002) 185:1644-9. doi: 10.1086/ 340574

32. King CH, Magak P, Salam EA, Ouma JH, Kariuki HC, Blanton RE. Measuring morbidity in schistosomiasis mansoni: relationship between image pattern, portal vein diameter and portal branch thickness in large-scale surveys using new WHO coding guidelines for ultrasound in schistosomiasis. Trop Med Int Health (2003) 8:109-17. doi: 10.1046/j.1365-3156.2003.00994.x

33. Kardorff R, Traore M, Diarra A, Sacko M, Maiga M, Franke D, et al. Lack of ultrasonographic evidence for severe hepatosplenic morbidity in schistosomiasis mansoni in Mali. Am J Trop Med Hyg. (1994) 51:190-7. doi: 10.4269/ajtmh.1994.51.190

34. Homeida M, Ahmed S, Dafalla A, Suliman S, Eltom I, Nash T, et al. Morbidity associated with Schistosoma mansoni infection as determined by ultrasound: a study in Gezira, Sudan. Am J Trop Med Hyg. (1988) 39:196-201. doi: 10.4269/ajtmh.1988.39.196

35. Mohamed-Ali Q, Elwali NE, Abdelhameed AA, Mergani A, Rahoud S, Elagib KE, et al. Susceptibility to periportal (Symmers) fibrosis in human Schistosoma mansoni infections: evidence that intensity and duration of infection, gender, and inherited factors are critical in disease progression. $J$ Infect Dis. (1999) 180:1298-306. doi: 10.1086/314999

36. Dessein AJ, Hillaire D, Elwali NE, Marquet S, Mohamed-Ali Q, Mirghani A, et al. Severe hepatic fibrosis in Schistosoma mansoni infection is controlled by a major locus that is closely linked to the interferon-gamma receptor gene. Am J Hum Genet. (1999) 65:709-21. doi: 10.1086/302526

37. Chevillard C, Moukoko CE, Elwali NE, Bream JH, Kouriba B, Argiro L, et al. IFN-gamma polymorphisms (IFN-gamma +2109 and IFN-gamma +3810 ) are associated with severe hepatic fibrosis in human hepatic schistosomiasis (Schistosoma mansoni). J Immunol. (2003) 171:5596-601. doi: 10.4049/jimmunol.171.10.5596

38. Abel L, Marquet S, Chevillard C, elWali NE, Hillaire D, Dessein A. [Genetic predisposition to bilharziasis in humans: research methods and application to the study of Schistosoma mansoni infection]. J Soc Biol. (2000) 194:15-8. doi: 10.1051/jbio/2000194010015

39. Dessein A, Chevillard C, Arnaud V, Hou X, Hamdoun AA, Dessein H, et al. Variants of CTGF are associated with hepatic fibrosis in Chinese, Sudanese, and Brazilians infected with schistosomes. J Exp Med. (2009) 206:2321-8. doi: 10.1084/jem.20090383 
40. Arnaud V, Li J, Wang Y, Fu X, Mengzhi S, Luo X, et al. Regulatory role of interleukin-10 and interferon-gamma in severe hepatic central and peripheral fibrosis in humans infected with Schistosoma japonicum. J Infect Dis. (2008) 198:418-26. doi: 10.1086/588826

41. Kouriba B, Chevillard C, Bream JH, Argiro L, Dessein H, Arnaud V, et al. Analysis of the 5q31-q33 locus shows an association between IL13-1055C/T IL-13-591A/G polymorphisms and Schistosoma haematobium infections. J Immunol. (2005) 174:6274-81. doi: 10.4049/jimmunol.174.10.6274

42. de Jesus AR, Magalhaes A, Miranda DG, Miranda RG, Araujo MI, de Jesus AA, et al. Association of type 2 cytokines with hepatic fibrosis in human Schistosoma mansoni infection. Infect Immun. (2004) 72:3391-7. doi: 10.1128/IAI.72.6.3391-3397.2004

43. Nono JK, Kamdem SD, Netongo PM, Dabee S, Schomaker M, Oumarou A, et al. Schistosomiasis burden and its association with lower measles vaccine responses in school children from rural Cameroon. Front Immunol. (2018) 9:2295. doi: 10.3389/fimmu.2018.02295

44. Oliveira JB, Silva PC, Vasconcelos LM, Gomes AV, Coelho MR, Cahu GG, et al. Influence of polymorphism (-G308A) TNF-alpha on the periportal fibrosis regression of schistosomiasis after specific treatment. Genet Test Mol Biomarke. (2015) 19:598-603. doi: 10.1089/gtmb.2015.0091

45. Silva PC, Gomes AV, de Souza TK, Coelho MR, Cahu GG, Muniz MT, et al. Association of SNP (-G1082A) IL-10 with increase in severity of periportal fibrosis in schistosomiasis, in the northeast of Brazil. Genet Test Mol Biomarke. (2014) 18:646-52. doi: 10.1089/gtmb.2014.0098

46. Alves Oliveira LF, Moreno EC, Gazzinelli G, Martins-Filho OA, Silveira AM, Gazzinelli A, et al. Cytokine production associated with periportal fibrosis during chronic schistosomiasis mansoni in humans. Infect Immun. (2006) 74:1215-21. doi: 10.1128/IAI.74.2.1215-1221.2006

47. Magalhaes A, Miranda DG, Miranda RG, Araujo MI, Jesus AA, Silva A, et al. Cytokine profile associated with human chronic schistosomiasis mansoni. Mem Inst Oswaldo Cruz (2004) 99(Suppl. 1):21-6. doi: 10.1590/S0074-02762004000900004

48. Coutinho HM, Acosta LP, Wu HW, McGarvey ST, Su L, Langdon GC, et al. Th2 cytokines are associated with persistent hepatic fibrosis in human Schistosoma japonicum infection. J Infect Dis. (2007) 195:288-95. doi: $10.1086 / 510313$

49. Silveira-Lemos D, Teixeira-Carvalho A, Martins-Filho OA, Alves Oliveira LF, Costa-Silva MF, Matoso LF, et al. Eosinophil activation status, cytokines and liver fibrosis in Schistosoma mansoni infected patients. Acta tropica. (2008) 108:150-9. doi: 10.1016/j.actatropica.2008.04.006

50. Long X, Chen Q, Zhao J, Rafaels N, Mathias $\mathrm{P}$, Liang $\mathrm{H}$, et al. An IL-13 promoter polymorphism associated with liver fibrosis in patients with Schistosoma japonicum. PLoS ONE (2015) 10:e0135360. doi: 10.1371/journal.pone.0135360

51. Mutengo MM, Mduluza T, Kelly P, Mwansa JC, Kwenda G, Musonda P, et al. Low IL-6, IL-10, and TNF- $\alpha$ and high IL-13 cytokine levels are associated with severe hepatic fibrosis in Schistosoma mansoni chronically exposed individuals. J Parasitol Res. (2018) 2018:9754060. doi: 10.1155/2018/9754060

52. Long X, Daya M, Zhao J, Rafaels N, Liang H, Potee J, et al. The role of ST2 and ST2 genetic variants in schistosomiasis. J Allergy Clin Immunol. (2017) 140:1416-22 e6. doi: 10.1016/j.jaci.2016.12.969

53. Zhang LW, Sun YL, Cai WM, Wang MH, He NX. [The activity of tumor necrosis factor in schistosomiasis japonica]. Zhongguo Ji Sheng Chong Xue Yu Ji Sheng Chong Bing Za Zhi. (1992) 10:11-3.

54. Jeng JE, Tsai JF, Chuang LY, Ho MS, Lin ZY, Hsieh MY, et al. Tumor necrosis factor-alpha 308.2 polymorphism is associated with advanced hepatic fibrosis and higher risk for hepatocellular carcinoma. Neoplasia. (2007) 9:987-92. doi: 10.1593/neo.07781

55. Moukoko CE, El Wali N, Saeed OK, Mohamed-Ali Q, Gaudart J, Dessein AJ, et al. No evidence for a major effect of tumor necrosis factor alpha gene polymorphisms in periportal fibrosis caused by Schistosoma mansoni infection. Infect Immun. (2003) 71:5456-60. doi: 10.1128/IAI.71.10.5456-5460.2003

56. Souza PR, Souza AL, Negrao-Correa D, Teixeira AL, Teixeira MM. The role of chemokines in controlling granulomatous inflammation in Schistosoma mansoni infection. Acta tropica. (2008) 108:135-8. doi: 10.1016/j.actatropica.2008.04.016

57. Rodrigues Oliveira JL, Teixeira MM, Lambertucci JR, Antunes CMF, Carneiro M, Negrao-Correa D. Plasma levels of innate immune mediators are associated with liver fibrosis in low parasite burden Schistosoma mansoni-infected individuals. Scand J Immunol. (2018) 87:e12642. doi: $10.1111 /$ sji.12642

58. Brigstock DR. Regulation of angiogenesis and endothelial cell function by connective tissue growth factor (CTGF) and cysteine-rich 61 (CYR61). Angiogenesis (2002) 5:153-65. doi: 10.1023/A:1023823803510

59. Shimo T, Nakanishi T, Nishida T, Asano M, Sasaki A, Kanyama M, et al. Involvement of CTGF, a hypertrophic chondrocyte-specific gene product, in tumor angiogenesis. Oncology (2001) 61:315-22. doi: 10.1159/000055339

60. Dessein A, Arnaud V, He H, Li J, Dessein H, Hou X, et al. Genetic analysis of human predisposition to hepatosplenic disease caused by schistosomes reveals the crucial role of connective tissue growth factor in rapid progression to severe hepatic fibrosis. Pathol Biol. (2013) 61:3-10. doi: 10.1016/j.patbio.2013.01.005

61. Li L, Wu T, Huang J, Ma K, Xu L, Wang H, et al. Expression of heat shock protein 47 , transforming growth factor-beta 1 , and connective tissue growth factor in liver tissue of patients with Schistosoma japonicum-induced hepatic fibrosis. Parasitology (2015) 142:341-51. doi: 10.1017/S0031182014001115

62. Tawfeek GM, Alafifi AM, Azmy MF. Immunological indicators of morbidity in human schistosomiasis mansoni: role of vascular endothelial growth factor and anti-soluble egg antigen IgG4 in disease progression. $J$ Egypt Soc Parasitol. (2003) 33:597-614.

63. de Toledo CF, Carvente CT, Shigueoka DC, Borges DR. Endothelial markers in schistosomiasis patients with or without portal hypertension. Dig Dis Sci. (2009) 54:1331-6. doi: 10.1007/s10620-008-0475-8

64. Pereira TA, Xie G, Choi SS, Syn WK, Voieta I, Lu J, et al. Macrophage-derived Hedgehog ligands promotes fibrogenic and angiogenic responses in human schistosomiasis mansoni. Liver Int. (2013) 33:149-61. doi: 10.1111/liv.12016

65. Pereira TA, Syn WK, Machado MV, Vidigal PV, Resende V, Voieta I, et al. Schistosome-induced cholangiocyte proliferation and osteopontin secretion correlate with fibrosis and portal hypertension in human and murine schistosomiasis mansoni. Clin Sci. (2015) 129:875-83. doi: 10.1042/CS20150117

66. Pereira TA, Syn WK, Amancio FF, Cunha PH, Caporali JF, Trindade $\mathrm{GV}$, et al. Osteopontin is upregulated in human and murine acute schistosomiasis mansoni. PLoS Negl Trop Dis. (2016) 10:e0005057. doi: 10.1371/journal.pntd.0005057

67. Bruha R, Jachymova M, Petrtyl J, Dvorak K, Lenicek M, Urbanek P, et al. Osteopontin: a non-invasive parameter of portal hypertension and prognostic marker of cirrhosis. World J Gastroenterol. (2016) 22:3441. doi: 10.3748/wjg.v22.i12.3441

68. Silveira AM, Bethony J, Gazzinelli A, Kloos H, de Oliveira Fraga LA, Alvares $\mathrm{MCB}$, et al. High levels of IgG4 to Schistosoma mansoni egg antigens in individuals with periportal fibrosis. Am J Trop Med Hyg. (2002) 66:542-9. doi: 10.4269/ajtmh.2002.66.542

69. Bonnard P, Remoue F, Schacht A-M, Deuffic-Burban S, Dompnier J-P, Elguero E, et al. Specific isotype immune response in the diagnosis of human schistosomiasis pathology? Am J Trop Med Hyg. (2004) 71:202-5. doi: 10.4269/ajtmh.2004.71.202

70. Negrão-Corrêa D, Fittipaldi JF, Lambertucci JR, Teixeira MM, de Figueiredo Antunes CM, Carneiro M. Association of Schistosoma mansoni-specific IgG and IgE antibody production and clinical schistosomiasis status in a rural area of Minas Gerais, Brazil. PLoS ONE (2014) 9:e88042. doi: 10.1371/journal.pone.0088042

71. Boctor F, Peter J. IgG subclasses in human chronic schistosomiasis: over-production of schistosome-specific and non-specific IgG4. Clin Exp Immunol. (1990) 82:574-8. doi: 10.1111/j.1365-2249.1990. tb05492.x

72. Eriksson J, Reimert CM, Kabatereine NB, Kazibwe F, Ireri E, Kadzo $\mathrm{H}$, et al. The $434(\mathrm{G}>\mathrm{C})$ polymorphism within the coding sequence of Eosinophil Cationic Protein (ECP) correlates with the natural course of Schistosoma mansoni infection. Int J Parasitol. (2007) 37:1359-66. doi: 10.1016/j.ijpara.2007.04.001

73. Silva PC, Gomes AV, Cahu GG, Coelho MR, Muniz MT, Domingues AL. Evaluation of the cytokine mannose-binding lectin as a mediator of periportal fibrosis progression in patients with schistosomiasis. Rev Soc Bras Med Trop. (2015) 48:350-3. doi: 10.1590/0037-8682-0309-2014

74. Cabantous S, Hou X, Louis L, He H, Mariani O, Sastre X, et al. Evidence for an important role of host microRNAs in regulating hepatic fibrosis in humans infected with Schistosoma japonicum. Int J Parasitol. (2017) 47:823-30. doi: 10.1016/j.ijpara.2017.05.007 
75. Vicentino AR, Carneiro VC, Allonso D, de Freitas Guilherme R, Benjamim CF, dos Santos HA, et al. Emerging role of HMGB1 in the pathogenesis of schistosomiasis liver fibrosis. Front Immunol. (2018) 9:1979. doi: 10.3389/fimmu.2018.01979

76. Hirayama $\mathrm{K}$, Chen $\mathrm{H}$, Kikuchi $\mathrm{M}$, Yin $\mathrm{T}, \mathrm{Gu} \mathrm{X}$, Liu J, et al. HLADR-DQ alleles and HLA-DP alleles are independently associated with susceptibility to different stages of post-schistosomal hepatic fibrosis in the Chinese population. Tissue Antigen. (1999) 53:269-74. doi: 10.1034/j.1399-0039.1999.530307.x

77. Das D, Singh NP, Kaur IR. Infectious Diseases and Their HLA Association. New Delhi: Editorial Committee.25 (2013).

78. Hirayama $\mathrm{K}$, Chen $\mathrm{H}$, Kikuchi $\mathrm{M}$, Yin $\mathrm{T}$, Itoh $\mathrm{M}, \mathrm{Gu} \mathrm{X}$, et al. Glycinevaline dimorphism at the 86th amino acid of HLA-DRB1 influenced the prognosis of postschistosomal hepatic fibrosis. J Infect Dis. (1998) 177:16826. doi: 10.1086/515299

79. Romano A, Hou X, Sertorio M, Dessein H, Cabantous S, Oliveira P, et al. Correction: FOXP3 + regulatory T cells in hepatic fibrosis and splenomegaly caused by Schistosoma japonicum: the spleen may be a major source of tregs in subjects with splenomegaly. PLoS Neglect Trop Dis. (2016) 10:e0004454. doi: 10.1371/journal.pntd.0004454

80. Pearce EJ, MacDonald AS. The immunobiology of schistosomiasis. Nat Rev Immunol. (2002) 2:499-511. doi: 10.1038/nri843

81. Nono JK, Ndlovu H, Aziz NA, Mpotje T, Hlaka L, Brombacher F. Host regulation of liver fibroproliferative pathology during experimental schistosomiasis via interleukin-4 receptor alpha. PLoS Neglect Trop Dis. (2017) 11:e0005861. doi: 10.1371/journal.pntd.0005861

82. Fallon PG, Mangan NE. Suppression of TH2-type allergic reactions by helminth infection. Nat Rev Immunol. (2007) 7:220-30. doi: 10.1038/nri2039

83. Mallat A, Preaux AM, Blazejewski S, Rosenbaum J, Dhumeaux D, Mavier P. Interferon alfa and gamma inhibit proliferation and collagen synthesis of human Ito cells in culture. Hepatology (1995) 21:1003-10. doi: 10.1002/hep.1840210418

84. Kovalovich K, DeAngelis RA, Li W, Furth EE, Ciliberto G, Taub R. Increased toxin-induced liver injury and fibrosis in interleukin-6-deficient mice. Hepatology (2000) 31:149-59. doi: 10.1002/hep.510310123

85. Nasir GA, Mohsin S, Khan M, Shams S, Ali G, Khan SN, et al. Mesenchymal stem cells and interleukin-6 attenuate liver fibrosis in mice. J Trans Med. (2013) 11:78. doi: 10.1186/1479-5876-11-78

86. Asai K, Hiki N, Mimura Y, Ogawa T, Unou K, Kaminishi M. Gender differences in cytokine secretion by human peripheral blood mononuclear cells: role of estrogen in modulating LPS-induced cytokine secretion in an ex vivo septic model. Shock (2001) 16:340-3. doi: 10.1097/00024382-200116050-00003

87. Guzdek A, Rokita H, Cichy J, Allison AC, Koj A. Rooperol tetraacetate decreases cytokine mRNA levels and binding capacity of transcription factors in U937 cells. Mediators Inflamm. (1998) 7:13-8. doi: 10.1080/09629359891324

88. Bataller R, North KE, Brenner DA. Genetic polymorphisms and the progression of liver fibrosis: a critical appraisal. Hepatology (2003) 37:493503. doi: 10.1053/jhep.2003.50127

89. Charo IF, Ransohoff RM. The many roles of chemokines and chemokine receptors in inflammation. N Engl J Med. (2006) 354:610-21. doi: 10.1056/NEJMra052723

90. Souza AL, Roffe E, Pinho V, Souza DG, Silva AF, Russo RC, et al. Potential role of the chemokine macrophage inflammatory protein 1alpha in human and experimental schistosomiasis. Infect Immun. (2005) 73:2515-23. doi: 10.1128/IAI.73.4.2515-2523.2005

91. Deshauer C, Morgan AM, Ryan EO, Handel TM, Prestegard JH, Wang $\mathrm{X}$. Interactions of the chemokine CCL5/RANTES with mediumsized chondroitin sulfate ligands. Structure (2015) 23:1066-77. doi: 10.1016/j.str.2015.03.024

92. Colley DG, Bustinduy AL, Secor WE, King CH. Human schistosomiasis. Lancet (2014) 383:2253-64. doi: 10.1016/S0140-6736(13)61949-2

93. Syn WK, Choi SS, Liaskou E, Karaca GF, Agboola KM, Oo YH, et al. Osteopontin is induced by hedgehog pathway activation and promotes fibrosis progression in nonalcoholic steatohepatitis. Hepatology (2011) 53:106-15. doi: 10.1002/hep.23998
94. Wen Y, Jeong S, Xia Q, Kong X. Role of osteopontin in liver diseases. Int J Biol Sci. (2016) 12:1121-8. doi: 10.7150/ijbs.16445

95. Butterworth AE, Wassom DL, Gleich GJ, Loegering DA, David JR. Damage to schistosomula of Schistosoma mansoni induced directly by eosinophil major basic protein. J Immunol. (1979) 122:221-9.

96. Rubin J, Zagai U, Blom K, Trulson A, Engström Å, Venge P. The coding ECP $434(\mathrm{G}>\mathrm{C})$ gene polymorphism determines the cytotoxicity of ECP but has minor effects on fibroblast-mediated gel contraction and no effect on RNase activity. J Immunol. (2009) 183:445-51. doi: 10.4049/jimmunol.0803912

97. Masterson JC, Capocelli KE, Hosford L, Biette K, McNamee EN, De Zoeten EF, et al. Eosinophils and IL-33 perpetuate chronic inflammation and fibrosis in a pediatric population with stricturing Crohn's ileitis. Inflamm Bowel Dis. (2015) 21:2429-40. doi: 10.1097/MIB.0000000000000512

98. Wong DT, Donoff RB, Yang J, Song B-Z, Matossian K, Nagura N, et al. Sequential expression of transforming growth factors $\alpha$ and $\beta 1$ by eosinophils during cutaneous wound healing in the hamster. Am J Pathol. (1993) 143:130-42.

99. Levi-Schaffer F, Garbuzenko E, Rubin A, Reich R, Pickholz D, Gillery P, et al. Human eosinophils regulate human lung-and skin-derived fibroblast properties in vitro: a role for transforming growth factor $\beta$ (TGF- $\beta$ ). Proc Natl Acad Sci USA. (1999) 96:9660-5. doi: 10.1073/pnas.96.17.9660

100. Brown K, Keogh M, Tagiuri N, Grainge M, Presanis J, Ryder S, et al. Severe fibrosis in hepatitis $\mathrm{C}$ virus-infected patients is associated with increased activity of the mannan-binding lectin (MBL)/MBL-associated serine protease 1 (MASP-1) complex. Clin Exp Immunol. (2007) 147:90-8. doi: $10.1111 /$ j.1365-2249.2006.03264.x

101. Ogawa T, Enomoto M, Fujii H, Sekiya Y, Yoshizato K, Ikeda K, et al. MicroRNA-221/222 upregulation indicates the activation of stellate cells and the progression of liver fibrosis. Gut (2012) 61:1600-9. doi: 10.1136/gutjnl-2011-300717

102. Zhou G, Lin W, Fang P, Lin X, Zhuge L, Hu Z, et al. MiR-10a improves hepatic fibrosis by regulating the TGF $\beta 1 /$ Smads signal transduction pathway. Exp Ther Med. (2016) 12:1719-22. doi: 10.3892/etm.2016.3542

103. Cai P, Piao X, Liu S, Hou N, Wang H, Chen Q. MicroRNA-gene expression network in murine liver during Schistosoma japonicum infection. PLoS ONE (2013) 8:e67037. doi: 10.1371/journal.pone.0067037

104. He X, Sun Y, Lei N, Fan X, Zhang C, Wang Y, et al. MicroRNA-351 promotes schistosomiasis-induced hepatic fibrosis by targeting the vitamin $\mathrm{D}$ receptor. Proc Natl Acad Sci USA. (2018) 115:180-5. doi: 10.1073/pnas.1715965115

105. Watanabe K, Mwinzi PN, Black CL, Muok EM, Karanja DM, Secor WE, et al. T regulatory cell levels decrease in people infected with Schistosoma mansoni on effective treatment. Am J Trop Med Hyg. (2007) 77:676-82. doi: 10.4269/ajtmh.2007.77.676

106. Gerlitz G, Hock R, Ueda T, Bustin M. The dynamics of HMG proteinchromatin interactions in living cells. Biochem Cell Biol. (2009) 87:127-37. doi: 10.1139/O08-110

107. Li LC, Gao J, Li J. Emerging role of HMGB 1 in fibrotic diseases. J Cell Mol Med. (2014) 18:2331-9. doi: 10.1111/jcmm.12419

108. Albayrak A, Uyanik MH, Cerrah S, Altas S, Dursun H, Demir M, et al. Is HMGB1 a new indirect marker for revealing fibrosis in chronic hepatitis and a new therapeutic target in treatment? Viral Immunol. (2010) 23:633-8. doi: 10.1089/vim.2010.0080

109. Kao Y-H, Jawan B, Goto S, Hung C-T, Lin Y-C, Nakano T, et al. (eds). Highmobility group box 1 protein activates hepatic stellate cells in vitro. Transpl Proc. (2008) 40:2704-5. doi: 10.1016/j.transproceed.2008.07.055

Conflict of Interest Statement: The authors declare that the research was conducted in the absence of any commercial or financial relationships that could be construed as a potential conflict of interest.

Copyright (C) 2018 Kamdem, Moyou-Somo, Brombacher and Nono. This is an openaccess article distributed under the terms of the Creative Commons Attribution License (CC BY). The use, distribution or reproduction in other forums is permitted, provided the original author(s) and the copyright owner(s) are credited and that the original publication in this journal is cited, in accordance with accepted academic practice. No use, distribution or reproduction is permitted which does not comply with these terms. 